B cells of multiple sclerosis patients induce autoreactive proinflammatory $\mathrm{T}$ cell responses

Peer-reviewed author version

FRAUSSEN, Judith; CLAES, Nele; VAN WIJMEERSCH, Bart; VAN HORSSEN, Jack; STINISSEN, Piet; Hupperts, Raymond \& SOMERS, Veerle (2016) B cells of multiple sclerosis patients induce autoreactive proinflammatory $T$ cell responses. In: CLINICAL IMMUNOLOGY, 173, p. 124-132.

DOI: 10.1016/j.clim.2016.10.001

Handle: http://hdl.handle.net/1942/23270 


\section{B cells of multiple sclerosis patients induce autoreactive proinflammatory}

\section{T cell responses}

Judith $\underline{\text { Fraussen }}^{\mathrm{a}^{*}}$, Nele $\underline{\text { Claes }}^{\mathrm{a}^{*}}$, Bart $\underline{\text { Van Wiimeersch }}^{\mathrm{a}, \mathrm{b}}$, Jack van Horssen ${ }^{\mathrm{a}, \mathrm{c}}$, Piet $\underline{\text { Stinissen }}^{\mathrm{a}}$, Raymond

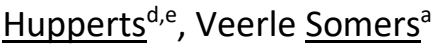

* These authors contributed equally

a Hasselt University, Biomedical Research Institute and Transnationale Universiteit Limburg, School of Life Sciences, Diepenbeek, Belgium

b Revalidation \& MS Center, Overpelt, Belgium

c Department of Molecular Cell Biology and Immunology, VU University Medical Center, Amsterdam, the Netherlands

d Department of Neuroscience, School of Mental Health and Neuroscience, Maastricht University, Maastricht, The Netherlands

e Academic MS Center Limburg, Zuyderland Medisch Centrum, Sittard, The Netherlands

Corresponding author: Veerle Somers, Hasselt University, Biomedical Research Institute, and Transnationale Universiteit Limburg, School of Life Sciences, Martelarenlaan 42, 3500 Hasselt, Belgium, Tel: +32(0)11269202, Fax: +32(0)11269299, E-mail: veerle.somers@uhasselt.be 


\section{Abstract}

Antibody-independent B cell functions play an important role in multiple sclerosis (MS) pathogenesis. In this study, B cell antigen presentation and costimulation in MS were studied. Peripheral blood B cells of MS patients showed increased expression of costimulatory CD86 and CD80 molecules compared with healthy controls (HC). In MS cerebrospinal fluid (CSF), 12-fold and 2-fold increases in $\mathrm{CD}^{+} 6^{+}$and $\mathrm{CD} 80^{+} \mathrm{B}$ cells, respectively, were evidenced compared with peripheral blood. Further, $\mathrm{B}$ cells from MS patients induced proinflammatory $T$ cells in response to myelin basic protein (MBP). Immunomodulatory treatment restored B cell costimulatory molecule expression and caused significantly reduced B cell induced T cell responses. Together, these results demonstrate the potential of $B$ cells from MS patients to induce autoreactive proinflammatory $T$ cell responses. Immunomodulatory therapy abrogated this effect, emphasizing the importance of B cell antigen presentation and costimulation in MS pathology.

Keywords: multiple sclerosis, B cells, antigen presentation, costimulatory molecules, T cells

List of abbreviations: $A P C$, antigen presenting cell; $B C R, B$ cell receptor; $C M$, culture medium; $C M V$, cytomegalovirus; CNS, central nervous system; CSF, cerebrospinal fluid; EAE, experimental autoimmune encephalomyelitis; EDSS, expanded disability status scale; HC, healthy control; MBP, myelin basic protein; MOG, myelin oligodendrocyte glycoprotein; PE, phycoerythrin; PPMS, primary progressive MS; RRMS, relapsing-remitting MS; SI, stimulation index; SPMS, secondary progressive MS; $\mathrm{TT}$, tetanus toxoid, $\triangle \mathrm{PF}$, proliferating fraction 


\section{Introduction}

MS is an inflammatory demyelinating disease of the central nervous system (CNS). Different clinical subtypes exist, including relapsing-remitting MS (RRMS) in which clinical relapses alternate with remission (80-85\%), secondary progressive MS (SPMS) with progressive neurological disease in $90 \%$ of RRMS patients within 25 years and primary progressive MS (PPMS) with a progressive course from the onset (10-15\%) [1]. Research into the role of B cells in MS pathogenesis has mainly focused on the production of autoantibodies. Oligoclonal immunoglobulin bands in the cerebrospinal fluid (CSF) are used for diagnostic confirmation [2]. Several CNS autoantigens that are targeted by the humoral immune response in MS have been described, although disease specificity and pathologic potential of most of the reported autoantibodies is debatable [3]. Antibody-independent B cell functions have gained interest in regard to MS pathology due to the clinical benefit of the B cell depleting anti-CD20 monoclonal antibody rituximab in RRMS and more recently ocrelizumab in RRMS and even PPMS [4, 5]. As rituximab does not target antibody-secreting plasma cells, the observed decrease in gadoliniumenhancing lesions on magnetic resonance imaging (MRI) was not due to reduced serum and CSF antibody titers or altered oligoclonal antibodies $[6,7]$. Instead, the decrease in CSF T cell numbers and peripheral Th1 and Th17 responses following B cell depletion indicated the importance of B cells in T cell regulation in MS pathology $[6,8]$.

Antigen presentation and costimulation are important B cell functions in B-T cell interactions. B cells are professional antigen presenting cells (APC) that are highly efficient in the activation of antigenspecific $C D 4^{+} T$ cells. After $B$ cell receptor $(B C R)$ recognition and processing, antigens are presented on the surface of the B cells by MHC molecules. Moreover, B cells express costimulatory molecules, such as CD86 and CD80, that are necessary to obtain full APC potency [9]. Several in vitro studies have shown that human B cells can induce effective T cell responses towards both foreign and self antigens [10, 11]. In MS patients, peripheral $B$ cells showed increased expression of $C D 80$ and $M H C I$ during active disease [12]. Memory B cells from the peripheral blood of some RRMS patients induced autoreactive T cell proliferation and IFN- $\gamma$ production [13]. More information is available from studies using 
experimental autoimmune encephalomyelitis (EAE), in which B cells promoted the differentiation of myelin oligodendrocyte glycoprotein (MOG)-specific Th1 and Th17 cells [14]. Interestingly, mice selectively deficient in $\mathrm{MHCll}$ molecules on $\mathrm{B}$ cells were resistant to EAE induction and exhibited diminished Th1 and Th17 responses [15]. Moreover, B cell antigen presentation was proven to be crucial for maximal disease in EAE, further emphasizing the importance of $\mathrm{B}$ cells in driving $\mathrm{CD} 4^{+} \mathrm{T}$ cell autoreactivity [16].

In this study, we measured B cell expression of costimulatory and human leukocyte antigen (HLA) molecules in the peripheral blood, CSF and brain lesions of MS patients and controls and characterized the T cell response (Th1, Th2, Th17) induced by B cells presenting myelin and viral antigens. As the importance of B cell involvement in MS pathogenesis also supports therapeutic B cell targeting, we compared the B cell antigen presentation potential between untreated MS patients and MS patients receiving immunomodulatory treatment. Our findings provide novel insight into the role of B-T cell interactions in MS pathology and elucidate whether current therapies for MS also act on the antigen presentation potential of B cells. 


\section{Materials and methods}

\subsection{Human samples}

MS patients, diagnosed according to the McDonald criteria [17], and healthy controls (HC) were recruited at the Revalidation \& MS Center (Overpelt, Belgium), Zuyderland Medical Center (Sittard, The Netherlands) and Biomedical Research Institute (Diepenbeek, Belgium) after informed consent and the institutes' ethics committees' approval. Samples were stored in the University Biobank Limburg. Clinical data are provided in Table 1.

Flow cytometry was done using $52 \mathrm{HC}$ and $74 \mathrm{MS}$ patients, 41 untreated and 33 receiving immunomodulatory treatment, including IFN- $\beta(n=13)$, glatiramer acetate $(n=5)$, fingolimod $(n=7)$, natalizumab $(n=4)$ and others $(n=4)$. Paired CSF samples were collected from 20 out of 74 MS patients, of which 18 were untreated and 2 received IFN- $\beta$ treatment. B cell proliferation assay was done for 5 untreated MS patients and 6 age- and gender-matched HC. B:T coculture assay was performed for treated $(n=10)$ and untreated $(n=9)$ MS patients and matched $H C(n=10)$. Treatment included IFN- $\beta(n=4)$, dimethylfumarate $(n=3)$ and teriflunomide $(n=3)$.

\subsection{Cell isolation}

PBMC were isolated from whole blood by density gradient centrifugation (Lympholyte, Cedarlane Laboratories, Sanbio B.V., Uden, The Netherlands). Cells were collected by centrifugation of the CSF for $12 \mathrm{~min}$ at $250 \mathrm{~g}$. CSF samples contaminated with red blood cells were excluded from analyses.

B cells were purified from PBMC using magnetic negative (STEMCELL Technologies SARL, Grenoble, France) or positive (Miltenyi Biotec B.V., Leiden, The Netherlands) selection. T cells were enriched by magnetic negative selection (STEMCELL Technologies SARL). Purity was confirmed on a FACSAria II flow cytometer (BD Biosciences, Erembodegem, Belgium).

\subsection{Flow cytometric analysis}


PBMC or CSF cells were stained using the following anti-human fluorescein isothiocyanate (FITC)-, phycoerythrin (PE)- or peridinin chlorophyll protein (PerCP)-labelled monoclonal antibodies: CD19, HLA-DR/DP/DQ, HLA-A/B/C, CD86, CD80 or CD40 (all from BD Biosciences). Isotype controls were used for gating strategies (BD Biosciences). Samples were analysed on a FACSCalibur flow cytometer using CellQuest software (BD Biosciences).

\subsection{Immunohistochemistry}

Formalin-fixed paraffin-embedded sections from MS brain tissue ( $n=4$, Netherlands Brain Bank) were deparaffinized and rehydrated. Following antigen retrieval in citrate buffer $\mathrm{pH} 6.0$, sections were stained overnight at $4^{\circ} \mathrm{C}$ with anti-CD20 (1:100, Dako, Heverlee, Belgium) and $1 \mathrm{~h}$ at room temperature with anti-HLA-DR/DP/DQ (1:100, Dako) or anti-CD80 (1:100, Abcam, Cambridge, UK). After washing, sections were incubated with appropriate secondary antibodies (Life Technologies, Gent, Belgium) for 1h at room temperature. Antibodies were diluted in PBS/1\% BSA. Cell nuclei were labelled with DAPI and autofluorescence was blocked by $0.1 \%$ Sudan Black in $70 \%$ ethanol. Stained sections were evaluated on a Nikon Eclipse 80i microscope using standard objectives and NIS Elements BR 3.10 software (Nikon).

\subsection{B cell proliferation assay}

CFSE-labelled ( $1 \mu \mathrm{M}$, Life Technologies) B cells were seeded at $1 \times 10^{5}$ cells with $1 \times 10^{5}$ autologous irradiated (8275 Rad) PBMC in triplicate. Culture medium (CM) was RPMI-1640 (Lonza, Verviers, Belgium) supplemented with $10 \%$ foetal bovine serum (FBS, Life Technologies), $1 \%$ sodium pyruvate, $1 \%$ nonessential amino acids, $50 \mathrm{U} / \mathrm{ml}$ penicillin and $50 \mathrm{\mu g} / \mathrm{ml}$ streptomycin (all from Sigma-Aldrich, Diegem, Belgium). For stimulation, 2,5 limit of flocculation (Lf)/ml tetanus toxoid (TT, RIVM, Bilthoven, The Netherlands), $1 \mu \mathrm{g} / \mathrm{ml}$ cytomegalovirus (CMV, BD Biosciences), $40 \mu \mathrm{g} / \mathrm{ml}$ human myelin basic protein (MBP, purified as described [18]) or $10 \mu \mathrm{g} / \mathrm{ml}$ human MOG peptides 1-22, 34-56, 64-86, 74-96 (Severn Biotech Ltd., Worchester, UK) were added. Unstimulated B cells or $1 \mu \mathrm{g} / \mathrm{ml} \mathrm{CpG2006}$ 
(Invivogen, Toulouse, France) with $50 \mathrm{U} / \mathrm{ml} \mathrm{IL-2} \mathrm{(Sigma-Aldrich)} \mathrm{were} \mathrm{used} \mathrm{as} \mathrm{a} \mathrm{negative} \mathrm{or} \mathrm{positive}$ control, respectively. After 13 days, B cell proliferation was assessed by flow cytometry using antihuman HLA-A/B/C peridinin chlorophyll protein (PerCP) (Biolegend, London, UK), 7-aminoactinomycin $D$ (7-AAD), HLA-DR PE and CD80 PE (all from BD Biosciences). The $\triangle$ proliferating fraction ( $\triangle P F, \%)$ was calculated by subtracting the mean background proliferation from the mean proliferation in response to antigen and was positive when $>2 \%$. Analysis was done on a FACSAria II cytometer and FACSDiva software (BD Biosciences).

\subsection{B:T coculture assay}

Purified B cells were loaded with $20 \mathrm{Lf} / \mathrm{ml} \mathrm{TT}, 100 \mu \mathrm{g} / \mathrm{ml} \mathrm{MBP,} 30 \mu \mathrm{g} / \mathrm{ml}$ MOG peptide 34-56 or 10 $\mu \mathrm{g} / \mathrm{ml} \mathrm{CMV} \mathrm{(Microbix,} \mathrm{Ontario,} \mathrm{Canada).} \mathrm{Loaded} \mathrm{or} \mathrm{non-loaded} \mathrm{irradiated} \mathrm{(1500} \mathrm{Rad)} \mathrm{B} \mathrm{cells} \mathrm{were}$ cocultured 2:1 with autologous CFSE-labelled T cells. T or B cells were cultured separately to analyse non-specific proliferation, which was absent in all tested samples. PHA (Sigma-Aldrich) was included as a positive control. MHCII:TCR interactions were blocked in cocultures with $\mathrm{TT}$ using $5 \mu \mathrm{g} / \mathrm{ml}$ azidefree anti-HLA-DR (BD Biosciences), anti-HLA-A/B/C or isotype control antibody (Biolegend) for 3 untreated MS patients, 2 treated MS patients and 5 HC. After 9 days, T cell proliferation was measured by flow cytometry using anti-human CD3 allophycocyanin-Cy7 (Biolegend), CD19 PE-Cy7, CD4 PECF594, CD8 PE, CD25 allophycocyanin and 7-AAD (all from BD Biosciences). Analysis was done on a FACSAria II flow cytometer using FACSDiva software (both from BD Biosciences). The $\triangle P F(\%)$ was calculated as described above. The stimulation index (SI) was calculated by dividing the mean proliferation in response to antigen by the mean background proliferation (CM). An antigenic T cell response was significant when $\Delta \mathrm{PF}>2 \%$ and $\mathrm{SI}>1.5$.

\subsection{Cytokine ELISA}

Production of IFN- $\gamma$, IL-4 and IL-17A in the B:T coculture assays was quantified using commercial ELISA (eBioscience, Vienna, Austria), following instructions of the manufacturer. Supernatants were diluted 
1:10 for IFN- $\gamma, 1: 2$ for IL-4 and 1:5 for IL-17A. Background OD (CM conditions) were subtracted from OD measured in the antigen-stimulated conditions.

\subsection{Statistics}

Statistical analyses were performed using Prism software (Graphpad). Comparison of multiple groups was done using ANOVA (Kruskal-Wallis) or Chi-square analysis of contingency tables. When comparing 2 groups, Mann-Whitney $U$ test or Fisher's Exact test were used. A p value $<0.05$ was considered statistically significant. 


\section{Results}

\subsection{B cells of MS patients show an increased expression of costimulatory molecules}

The ability of B cells to present (auto)antigens to T cells in MS patients was first studied by analysing the expression of antigen presentation (HLA) and costimulatory molecules on B cells from the peripheral blood, CSF and brain tissue of MS patients and controls. CD80, CD86, CD40 and HLA molecules are classical markers of B cell activation that work together to obtain full APC potency [9]. Nearly all peripheral B cells (> 90\%) showed expression of HLA-DR/DP/DQ, HLA-A/B/C and CD40, while $\mathrm{CD}^{+} 6^{+}(\sim 4 \%)$ and $\mathrm{CD} 80^{+}(\sim 19.5 \%)$ cells were less frequent in the peripheral B cell pool (Fig. 1A). The percentage of $\mathrm{CD} 86^{+}$and $\mathrm{CD} 80^{+} \mathrm{B}$ cells was significantly increased in the peripheral blood of $\mathrm{MS}$ patients $(4.46 \pm 0.39 \%$ and $21.15 \pm 1.30 \%$, respectively) when compared to $\mathrm{HC}(3.51 \pm 0.45 \%$ and $17.11 \pm 1.14 \%$, respectively, $p<0.05$, Fig. 1B). Also the expression levels, indicated by the mean fluorescence intensity (MFI), of CD80 and CD40 were significantly elevated on peripheral B cells of MS patients ( $24.87 \pm 0.41$ and $43.38 \pm 1.21$, respectively) in comparison with $\mathrm{HC}(23.81 \pm 0.54$ and $39.21 \pm 0.84$, respectively, $p<0.05$, Fig. $1 \mathrm{C})$. Interestingly, when dividing the MS population into untreated and treated patients, significantly increased costimulatory molecule expression was only present on peripheral B cells of untreated MS patients. Thus, the use of immunomodulatory treatment restored B cell expression of costimulatory molecules. No difference was observed in B cell expression of costimulatory and MHC molecules between the included treatments (Supplementary Fig. A.1). Although the MS population was older than the HC population, none of the observed effects was correlated with age (data not shown).

The observed increase in costimulatory molecule expression was even more pronounced on CSF B cells. The frequencies of $\mathrm{CD}^{+} 6^{+}(61.95 \pm 4.68 \%)$ and $\mathrm{CD}^{\circ} 0^{+}(54.84 \pm 4.61 \%) \mathrm{B}$ cells in MS CSF were increased 20-fold and 3-fold, respectively, compared to paired PBMC ( $p<0.001$, Fig. 2A-B). Moreover, we observed a 2-fold increase in the expression levels of CD86 $(66.81 \pm 4.93, p<0.001)$ and HLA-A/B/C (501.60 $\pm 91.34, p<0.01)$ on CSF B cells, while CD80 expression $(35.57 \pm 3.52, p<0.05)$ was elevated 1.5 
times compared to PBMC (Fig. 2C). This increased costimulatory molecule expression on CSF B cells could be due to differences in B cell subtype distribution (Supplementary Fig. A.2). Additionally, we found that the majority of infiltrating B cells in MS brain lesions expressed HLA-DR (Fig. 2D) and CD80 (Fig. 2E) when analysing post-mortem brain tissue.

These results indicate an increased costimulatory molecule expression on peripheral blood B cells of MS patients, which was further enhanced on CNS infiltrating B cells. Thus, B cells of MS patients might be more efficient in the presentation of (auto)antigens to T cells.

\subsection{B cells upregulate CD80 expression following antigen-specific stimulation}

B cells were previously shown to increase CD80 and HLA-DR expression following polyclonal or CD40 ligand (CD40L) stimulation [19]. Using a B cell proliferation assay, we investigated upregulation of costimulatory and HLA molecules on B cells following antigen-specific stimulation and determined the frequency of peripheral CMV- and autoreactive B cells in HC and MS patients. CFSE-labelled B cells from HC and MS patients were stimulated by CpG2006 (polyclonal activation, positive control), TT (control antigen), CMV, MBP or MOG. CMV was included as it shares a mimicry motif with MOG and induced MOG reactive T cells in an EAE rhesus monkey model [20]. B cell proliferation and expression (MFI) of CD80 and HLA molecules were measured after 13 days of culture.

An example of the output data highlighting the variability in responses between $\mathrm{CM}, \mathrm{TT}$ and polyclonal stimulation is given in Fig. 3A. The 3- to 4-fold increase in CD80 and HLA-DR expression on CpG2006 stimulated B cells confirmed the upregulation of costimulatory and antigen presentation molecules on B cells following polyclonal activation ( $p<0.05$, Fig. 3B). Antigen-specific proliferation ( $\triangle P F \geq 2$ ) caused a significant 2-fold upregulation of CD80 expression on the responding B cells ( $p<0.05$, Fig. 3B). Levels of HLA-DR and HLA-A/B/C expression on B cells remained unchanged following antigen-specific stimulation. $\mathrm{HC}$ and MS patients were combined as no difference was observed in B cell CD80 and HLA expression between these groups. These results suggest an increased potential of B cells to stimulate B:T cell interactions, not only following polyclonal but also following antigen-specific stimulation. 
When analysing the percentage of proliferating $\mathrm{B}$ cells against $\mathrm{TT}, \mathrm{CMV}, \mathrm{MBP}$ or MOG, a similar frequency of peripheral CMV- and autoreactive B cells was indicated in $\mathrm{HC}$ and MS patients (Fig. $3 \mathrm{C}$ ). The above results indicate that viral and autoantigen-specific B cells are present in the peripheral blood of both MS patients and HC. Following antigen-specific stimulation, these B cells upregulate CD80 expression, thereby further increasing their costimulatory capacity.

\subsection{B cells of MS patients induce MBP-specific proinflammatory T cell proliferation}

The next step was to functionally validate the antigen presentation potential of peripheral blood B cells from $\mathrm{HC}$, untreated and treated MS patients. Using a B:T coculture assay, we studied the capacity of B cells to induce T cell proliferation following antigen processing and presentation of the CNS-specific autoantigens MBP and MOG and the viral antigen CMV.

Examples of the output data from 2 representative MS patients and $1 \mathrm{HC}$ are shown in Fig. 4, while results from all performed assays are shown in Fig. 5. We first excluded differences in $T$ cell responsiveness in the 3 study groups by showing similar T cell proliferation ( $\triangle \mathrm{PF}$ ) towards PHA (Fig. 5A). B cells from HC, untreated and treated MS patients also elicited similar T cell proliferation against TT, CMV and MOG (Fig. 5B). For MBP, B cell induced T cell proliferation was observed in MS patients (2/9 untreated and $1 / 10$ treated MS patients) (Fig. 5B-C). Interestingly, the percentage of donors with positive B cell induced T cell proliferation towards TT and CMV was significantly decreased in the treated MS group compared with $\mathrm{HC}$ and untreated MS patients $(\mathrm{p}<0.05$, Fig. $5 \mathrm{C})$. Of the treated MS patients reacting towards TT and CMV, the majority (50\%) were treated with IFN- $\beta$, although B cells of MS patients treated with dimethylfumarate and teriflunomide also showed a trend to decreased CD80 and CD86 expression at day 0 of the coculture (data not shown). As a proof of concept, the necessity of $\mathrm{MHCl} / \mathrm{Il}$ in the observed T cell responses towards TT was indicated by addition of an anti-HLA-DR or anti-HLA-A/B/C antibody. Efficient blocking of both MHCII (HLA-DR, $p<0.001$ ) and MHCI (HLA-A/B/C, $\mathrm{p}<0.05)$ interactions with T cells in cocultures with TT was demonstrated, while an isotype control antibody had no effect (Fig. 5D-E). 
Next, we characterized the B cell induced T cell responses. Although the majority of proliferating $T$ cells were $\mathrm{CD}^{+}$, little $\mathrm{CD} 8^{+} \mathrm{T}$ cell proliferation was evidenced against all tested antigens in both $\mathrm{HC}$ and $\mathrm{MS}$ patients (Fig. 6A). The B cell induced $C D 4^{+}$Th cell phenotype was analysed by measuring the production of IFN- $\gamma$, IL-17A and IL-4 in proliferating conditions of B:T cocultures using ELISA (Fig. 6B). In PHAstimulated conditions, all tested cytokines were present while B or T cells cultured alone did not produce cytokines (data not shown). B cells of HC and MS patients (untreated and treated) mainly induced IFN- $\gamma$ producing Th1 cells towards all tested antigens. For untreated MS patients, observed B cell induced $\mathrm{T}$ cell responses against MBP were characterized by a high IFN- $\gamma$ production in all responding patients and IL-17A production in one patient. This corresponds with a proinflammatory Th1 and possibly Th17 phenotype. For treated MS patients, IFN- $\gamma$ production appeared to be decreased in comparison with untreated MS patients but this was not significant, probably because of the low number of positive patients. No Th2 cells were induced, as evidenced by the lack of IL-4 production. These results indicate that $\mathrm{B}$ cells of $\mathrm{HC}$, untreated and treated MS patients can induce T cell responses towards TT, CMV and MOG. In addition, MBP-specific B cell induced T cell proliferation was evidenced in MS patients. B cell induced responses consisted mainly of proinflammatory Th1 cells, although IL17A producing T cells were induced following B cell presentation of MBP in an untreated MS patient. Immunomodulatory treatment reduced $B$ cell induced $T$ cell proliferation against $T T$ and $C M V$. 


\section{Discussion}

The success of rituximab and ocrelizumab in the treatment of RRMS and even PPMS has renewed interest into antibody-independent B cell functions in MS pathogenesis [4-6, 8]. In this study, we demonstrated an increased expression of costimulatory molecules on peripheral blood B cells of MS patients, which was even more pronounced on CNS infiltrating B cells. Further, MS B cells induced MBP-specific proinflammatory Th1 responses. Interestingly, immunomodulatory treatment reduced the antigen presentation potential of MS B cells.

We observed a significant increase in the percentage of $\mathrm{CD} 86^{+}$and $\mathrm{CD} 80^{+} \mathrm{B}$ cells and the expression level of CD80 and CD40 on B cells from the peripheral blood of MS patients compared to HC. These observations are novel, as the increase in costimulatory molecules occurred irrespective of disease activity, which contrasts Genç et al. who found increased percentages of $\mathrm{CD}^{\circ} 0^{+} \mathrm{B}$ cells exclusively during MS exacerbation [12]. Increased CD40 expression on peripheral B cells has not yet been observed in MS patients up to now. CD80 is known to induce Th1 responses, leading to IFN- $\gamma$ and IL-2 production, while CD86 stimulates Th2 cells and results in IL-4 secretion and promotes humoral immunity [21]. Thus, our results point towards the involvement of both proinflammatory Th1 responses and autoantibodies in MS. CD80 and CD86 have further been implicated in the suppressive function of regulatory B cells (Bregs) together with IL-10 production [22]. Alternatively, Niino et al. showed that $C D 86^{+}$naïve B cells were increased in untreated MS patients, which suggests increased activation of resting T cells [23]. Thus, the increase in $\mathrm{CD}^{2} 6^{+} \mathrm{B}$ cells in our study could also be restricted to naïve B cells, pointing to the activation of resting T cells, possibly by epitope spreading or the release of antigens from the CNS.

The elevated B cell costimulatory molecule expression was only evident in untreated MS patients, indicating that immunomodulatory treatment can reduce B cell costimulatory molecule expression to levels seen in HC. This probably is an indirect effect by the induction of a shift towards an antiinflammatory immune profile $[24,25]$. IFN- $\beta$ treated MS patients were already shown to present with a decreased percentage of $\mathrm{CD} 80^{+}$and $\mathrm{CD} 86^{+} \mathrm{B}$ cells $[12,23]$. We now demonstrated that this decrease 
in B cell costimulatory molecule expression is not specific for IFN- $\beta$ treatment but is a more general finding in treated MS patients.

When analysing B cells infiltrating the CNS, we evidenced an even more pronounced increase in the frequency of $\mathrm{CD} 80^{+}$and $\mathrm{CD} 86^{+} \mathrm{B}$ cells in MS CSF compared to peripheral blood. Moreover, the expression level of CD80, CD86 and HLA-A/B/C was increased on CSF B cells, which suggests that CNSinfiltrating B cells are highly potent APC. Increased expression of costimulatory molecules on CSF B cells could be the result of the increased proportion of $\mathrm{CD} 19^{+}$plasmablasts in the CSF, indicating that these cells have a high antigen presentation capability. It is already known that memory B cells show higher expression levels of costimulatory molecules than naïve B cells [13]. The APC function of CNSinfiltrating B cells in MS patients was further underlined by the expression of CD80 and HLA-DR on the majority of B cells in perivascular infiltrates in MS brain lesions. Previously, a higher percentage of $\mathrm{CD} 80^{+}$CSF B cells from MS patients were indicated compared with neurological controls [26]. Further, our results were in agreement with Windhagen et al. who evidenced an increased CD80 expression on lymphocytes within perivascular infiltrates in acute MS lesions [27].

We next showed that B cells upregulate CD80 expression not only following polyclonal or CD40 ligand stimulation [19], but also following antigen-specific stimulation. Thus, B cells of MS patients express increased levels of costimulatory molecules that can be further elevated by autoantigen recognition, rendering them highly potent activators of autoreactive T cells. As expected, similar B cell proliferation against $\mathrm{TT}$ was shown in $\mathrm{HC}$ and MS patients. Myelin-specific B cells were present both in $\mathrm{HC}$ and MS patients, which is in agreement with a study by Harp et al. [13]. Also the frequency of MBP-reactive B cells is similar between both studies.

The antigen presentation potential of B cells from MS patients was then functionally evaluated using a B:T coculture assay. Interestingly, B cell induced T cell proliferation towards MBP was evidenced in a proportion of MS patients and not in $\mathrm{HC}$, although MBP-specific $B$ cells were equally present in $\mathrm{MS}$ patients and HC. Previously, the frequency of MBP-reactive T cells was also indicated to be equal in the peripheral blood of $\mathrm{HC}$ and MS patients [28]. Thus, although the number of included patients was 
limited, our results suggest that MBP-reactive T cells can only be stimulated by B cells in MS patients. This could be due to the increased costimulatory molecule expression on MS B cells. Screening of higher numbers of MS patients and $\mathrm{HC}$ is needed to further elucidate the true prevalence of these antigen-specific responses. The efficient blocking of B cell induced T cell proliferation to $T \mathrm{~T}$ by antiHLA-DR and anti-HLA-A/B/C antibodies emphasized the dependency on B cell antigen presentation. Further, the absence of differences in anti-MOG T cell responses induced by B cells between $\mathrm{HC}$ and MS patients could be explained by the use of MOG peptide, which bypasses the need of antigen processing.

T cells proliferating in response to MBP presented by B cells were mainly IFN- $\gamma$ producing Th1 cells. We also measured IL-17A in the supernatant of MBP-specific proliferating T cells from an untreated MS patient, indicating the possibility that MS B cells could also induce Th17 responses towards MBP. Th17 cells are a highly pathogenic $\mathrm{CD} 4^{+}$subtype that can induce and exacerbate disease in MS and EAE [29]. Moreover, our findings correlate with the decreased Th1 and Th17 responses that were observed following B cell depletion with rituximab $[6,8]$. T cell responses induced towards $\mathrm{CMV}$ and TT consisted mainly of IFN- $\gamma$, as expected [30, 31].

An interesting finding was the decrease in the number of treated MS patients positive for B cell induced T cell proliferation towards $T \mathrm{~T}$ and CMV. This is in agreement with the normal expression levels of costimulatory molecules on B cells from treated MS patients. Together, these results demonstrate that immunomodulatory treatment leads to a reduction in B cell induced proinflammatory $\mathrm{T}$ cell responses. As this effect is most pronouncedly seen for $T \mathrm{~T}$ and $\mathrm{CMV}$, it is probably due to a general reduction in $\mathrm{B}$ cell functionality.

Our results further underline the importance of B cells as regulators of $T$ cell responses in MS pathogenesis. The role of B cells in the activation of proinflammatory $T$ cells has long-time been neglected in MS, as B cells were not required for the generation of $\mathrm{T}$ cell responses to specific antigens in mice and were not essential contributors to EAE models based on peptide immunization [32]. Recent studies have highlighted the role of B cells as APC in EAE induction and pathology [14-16]. Further, 
clinical studies of rituximab in RRMS and ocrelizumab in RRMS and PPMS have increased the importance of B cell antigen presentation in MS. All these data point towards B cells as one of the driving immune cell subtypes in the initiation of the autoreactive T cell response in the MS CNS. Results from this study have shown that B cells of MS patients can induce autoreactive proinflammatory and pathogenic $T$ cell responses by their increased costimulatory molecule expression and high antigen presentation potential. Thus, following their escape from tolerance, $B$ cells could drive pathogenic $T$ cell responses in the initiation and progression of MS.

A limitation of this study is the number of included MS patients and HC for the functional assays. Due to the low frequency of $\mathrm{HC}$ and MS patients positive for MBP- or MOG-reactive B cells, the number of responding donors in the B:T coculture is low as well. The repertoire of autoantigens in MS is not restricted solely to myelin antigens, but also contains neuronal, oligodendrocyte and astrocyte antigens [3]. Future experiments should therefore include a higher number of possible target antigens of different cellular origins. In addition, the B cell subtype that plays a role in MS by means of antigen presentation has to be further determined. In this regard, both memory $B$ cells and $C D 11 c^{+} B$ cells were shown to possess high antigen presentation potential in autoimmunity [13, 33].

In conclusion, we have demonstrated the potential of peripheral B cells from MS patients to induce proinflammatory autoreactive T cell responses. These effects were reduced in MS patients under immunomodulatory treatment. Analysis of the B cell antigen presentation potential could lead to novel therapeutic opportunities for MS. 


\section{Acknowledgements}

We thank Kim Ulenaers, Igna Rutten, Anne Bogaers, Dr. Van Woensel (Hasselt University and UBiLim), Anita Knevels (Revalidation \& MS Center), Bertine Timmermans, Ingrid Mevissen-Smeets and Judith Poeth (Zuyderland Medical Center) for their help with collecting patient material and data.

\section{Funding}

This work was supported by Hasselt University and the Belgian Charcot Foundation. J. Fraussen is a postdoctoral fellow of the Fund for Scientific Research (FWO), Flanders. 


\section{References}

[1] H. Lassmann, W. Bruck, C.F. Lucchinetti, The immunopathology of multiple sclerosis: an overview, Brain. Pathol., 17 (2007) 210-218.

[2] S.R. Cole, R.W. Beck, P.S. Moke, D.I. Kaufman, W.W. Tourtellotte, The predictive value of CSF oligoclonal banding for MS 5 years after optic neuritis. Optic Neuritis Study Group, Neurology, $51(1998)$ 885-887.

[3] J. Fraussen, N. Claes, L. de Bock, V. Somers, Targets of the humoral autoimmune response in multiple sclerosis, Autoimmun. Rev, 13 (2014) 1126-1137.

[4] S.L. Hauser, E. Waubant, D.L. Arnold, T. Vollmer, J. Antel, R.J. Fox, A. Bar-Or, M. Panzara, N. Sarkar, S. Agarwal, A. Langer-Gould, C.H. Smith, B-cell depletion with rituximab in relapsingremitting multiple sclerosis, N. Engl. J Med, 358 (2008) 676-688.

[5] I. Fyfe, In the news: Ocrelizumab excites ectrims, Nat. Rev. Neurol., 11 (2015) 667.

[6] A.H. Cross, J.L. Stark, J. Lauber, M.J. Ramsbottom, J.A. Lyons, Rituximab reduces B cells and T cells in cerebrospinal fluid of multiple sclerosis patients, J. Neuroimmunol., 180 (2006) 63-70.

[7] M.d.P. Martin, P.D. Cravens, R. Winger, B.C. Kieseier, S. Cepok, T.N. Eagar, S.S. Zamvil, M.S. Weber, E.M. Frohman, B.K. Kleinschmidt-Demasters, T.J. Montine, B. Hemmer, C.M. Marra, O. Stuve, Depletion of B lymphocytes from cerebral perivascular spaces by rituximab, Arch. Neurol., 66 (2009) 1016-1020.

[8] A. Bar-Or, L. Fawaz, B. Fan, P.J. Darlington, A. Rieger, C. Ghorayeb, P.A. Calabresi, E. Waubant, S.L. Hauser, J. Zhang, C.H. Smith, Abnormal B-cell cytokine responses a trigger of T-cell-mediated disease in MS?, Ann. Neurol, 67 (2010) 452-461.

[9] A. Lanzavecchia, Antigen-specific interaction between T and B cells, Nature, 314 (1985) 537-539.

[10] C.L. Wilson, D.W. Hine, A. Pradipta, J.P. Pearson, E.W. van, J.H. Robinson, A.M. Knight, Presentation of the candidate rheumatoid arthritis autoantigen aggrecan by antigen-specific B cells induces enhanced CD4(+) T helper type 1 subset differentiation, Immunology, 135 (2012) 344-354. 
[11] E.G. Schmidt, N.N. Kristensen, M.H. Claesson, A.E. Pedersen, Enteroantigen-presenting B cells efficiently stimulate CD4(+) T cells in vitro, Inflamm. Bowel. Dis, 17 (2011) 308-318.

[12] K. Genc, D.L. Dona, A.T. Reder, Increased CD80(+) B cells in active multiple sclerosis and reversal by interferon beta-1b therapy, J. Clin. Invest, 99 (1997) 2664-2671.

[13] C.T. Harp, S. Ireland, L.S. Davis, G. Remington, B. Cassidy, P.D. Cravens, O. Stuve, A.E. LovettRacke, T.N. Eagar, B.M. Greenberg, M.K. Racke, L.G. Cowell, N.J. Karandikar, E.M. Frohman, N.L. Monson, Memory B cells from a subset of treatment-naive relapsing-remitting multiple sclerosis patients elicit CD4(+) T-cell proliferation and IFN-gamma production in response to myelin basic protein and myelin oligodendrocyte glycoprotein, Eur. J. Immunol, 40 (2010) 2942-2956.

[14] M.S. Weber, T. Prod'homme, J.C. Patarroyo, N. Molnarfi, T. Karnezis, K. Lehmann-Horn, D.M. Danilenko, J. Eastham-Anderson, A.J. Slavin, C. Linington, C.C. Bernard, F. Martin, S.S. Zamvil, Bcell activation influences T-cell polarization and outcome of anti-CD20 B-cell depletion in central nervous system autoimmunity, Ann. Neurol, 68 (2010) 369-383.

[15] N. Molnarfi, U. Schulze-Topphoff, M.S. Weber, J.C. Patarroyo, T. Prod'homme, M. Varrin-Doyer, A. Shetty, C. Linington, A.J. Slavin, J. Hidalgo, D.E. Jenne, H. Wekerle, R.A. Sobel, C.C. Bernard, M.J. Shlomchik, S.S. Zamvil, MHC class II-dependent B cell APC function is required for induction of CNS autoimmunity independent of myelin-specific antibodies, J. Exp. Med, 210 (2013) 29212937.

[16] C.R. Parker Harp, A.S. Archambault, J. Sim, S.T. Ferris, R.J. Mikesell, P.A. Koni, M. Shimoda, C. Linington, J.H. Russell, G.F. Wu, B cell antigen presentation is sufficient to drive neuroinflammation in an animal model of multiple sclerosis, J. Immunol, 194 (2015) 5077-5084.

[17] C.H. Polman, S.C. Reingold, B. Banwell, M. Clanet, J.A. Cohen, M. Filippi, K. Fujihara, E. Havrdova, M. Hutchinson, L. Kappos, F.D. Lublin, X. Montalban, P. O'Connor, M. Sandberg-Wollheim, A.J. Thompson, E. Waubant, B. Weinshenker, J.S. Wolinsky, Diagnostic criteria for multiple sclerosis: 2010 revisions to the McDonald criteria, Ann. Neurol, 69 (2011) 292-302. 
[18] G.E. Deibler, R.E. Martenson, M.W. Kies, Large scale preparation of myelin basic protein from central nervous tissue of several mammalian species, Prep. Biochem., 2 (1972) 139-165.

[19] C.T. Harp, A.E. Lovett-Racke, M.K. Racke, E.M. Frohman, N.L. Monson, Impact of myelin-specific antigen presenting B cells on T cell activation in multiple sclerosis, Clin. Immunol, 128 (2008) 382-391.

[20] H.P. Brok, L. Boven, M. van Meurs, d.R. Kerlero, L. Celebi-Paul, Y.S. Kap, A. Jagessar, R.Q. Hintzen, G. Keir, J. Bajramovic, A. Ben Nun, J. Bauer, J.D. Laman, S. Amor, B.A. 't Hart, The human CMVUL86 peptide 981-1003 shares a crossreactive T-cell epitope with the encephalitogenic MOG peptide 34-56, but lacks the capacity to induce EAE in rhesus monkeys, J. Neuroimmunol, 182 (2007) 135-152.

[21] V.K. Kuchroo, M.P. Das, J.A. Brown, A.M. Ranger, S.S. Zamvil, R.A. Sobel, H.L. Weiner, N. Nabavi, L.H. Glimcher, B7-1 and B7-2 costimulatory molecules activate differentially the Th1/Th2 developmental pathways: application to autoimmune disease therapy, Cell, 80 (1995) 707-718.

[22] P.A. Blair, L.Y. Norena, F. Flores-Borja, D.J. Rawlings, D.A. Isenberg, M.R. Ehrenstein, C. Mauri, CD19(+)CD24(hi)CD38(hi) B cells exhibit regulatory capacity in healthy individuals but are functionally impaired in systemic Lupus Erythematosus patients, Immunity, 32 (2010) 129-140.

[23] M. Niino, M. Hirotani, Y. Miyazaki, H. Sasaki, Memory and naive B-cell subsets in patients with multiple sclerosis, Neurosci. Lett, 464 (2009) 74-78.

[24] N. Claes, T. Dhaeze, J. Fraussen, B. Broux, B. Van Wijmeersch, P. Stinissen, R. Hupperts, N. Hellings, V. Somers, Compositional changes of B and T cell subtypes during fingolimod treatment in multiple sclerosis patients: a 12-month follow-up study, PLoS One, 9 (2014) e111115.

[25] R.D. Schubert, Y. Hu, G. Kumar, S. Szeto, P. Abraham, J. Winderl, J.M. Guthridge, G. Pardo, J. Dunn, L. Steinman, R.C. Axtell, IFN-beta treatment requires B cells for efficacy in neuroautoimmunity, J. Immunol., 194 (2015) 2110-2116.

[26] F. Sellebjerg, J. Jensen, L.P. Ryder, Costimulatory CD80 (B7-1) and CD86 (B7-2) on cerebrospinal fluid cells in multiple sclerosis, J. Neuroimmunol, 84 (1998) 179-187. 
[27] A. Windhagen, J. Newcombe, F. Dangond, C. Strand, M.N. Woodroofe, M.L. Cuzner, D.A. Hafler, Expression of costimulatory molecules B7-1 (CD80), B7-2 (CD86), and interleukin 12 cytokine in multiple sclerosis lesions, J. Exp. Med., 182 (1995) 1985-1996.

[28] N. Hellings, M. Baree, C. Verhoeven, B. D'Hooghe M, R. Medaer, C.C. Bernard, J. Raus, P. Stinissen, T-cell reactivity to multiple myelin antigens in multiple sclerosis patients and healthy controls, J. Neurosci. Res., 63 (2001) 290-302.

[29] L.E. Harrington, R.D. Hatton, P.R. Mangan, H. Turner, T.L. Murphy, K.M. Murphy, C.T. Weaver, Interleukin 17-producing CD4+ effector T cells develop via a lineage distinct from the Thelper type 1 and 2 lineages, Nat. Immunol, 6 (2005) 1123-1132.

[30] C. Cellerai, A. Harari, F. Vallelian, O. Boyman, G. Pantaleo, Functional and phenotypic characterization of tetanus toxoid-specific human CD4+ T cells following re-immunization, Eur. J. Immunol, 37 (2007) 1129-1138.

[31] P.J. van de Berg, K.M. Heutinck, R. Raabe, R.C. Minnee, S.L. Young, K.A. van Donselaar-van der Pant, F.J. Bemelman, R.A. van Lier, I.J. ten Berge, Human cytomegalovirus induces systemic immune activation characterized by a type 1 cytokine signature, J. Infect. Dis., 202 (2010) 690699.

[32] M.M. Epstein, F. Di Rosa, D. Jankovic, A. Sher, P. Matzinger, Successful T cell priming in B celldeficient mice, J. Exp. Med., 182 (1995) 915-922.

[33] A.V. Rubtsov, K. Rubtsova, J.W. Kappler, J. Jacobelli, R.S. Friedman, P. Marrack, CD11c-Expressing B Cells Are Located at the T Cell/B Cell Border in Spleen and Are Potent APCs, J. Immunol, 195 (2015) 71-79. 
Table 1. Characteristics of MS patients and HC

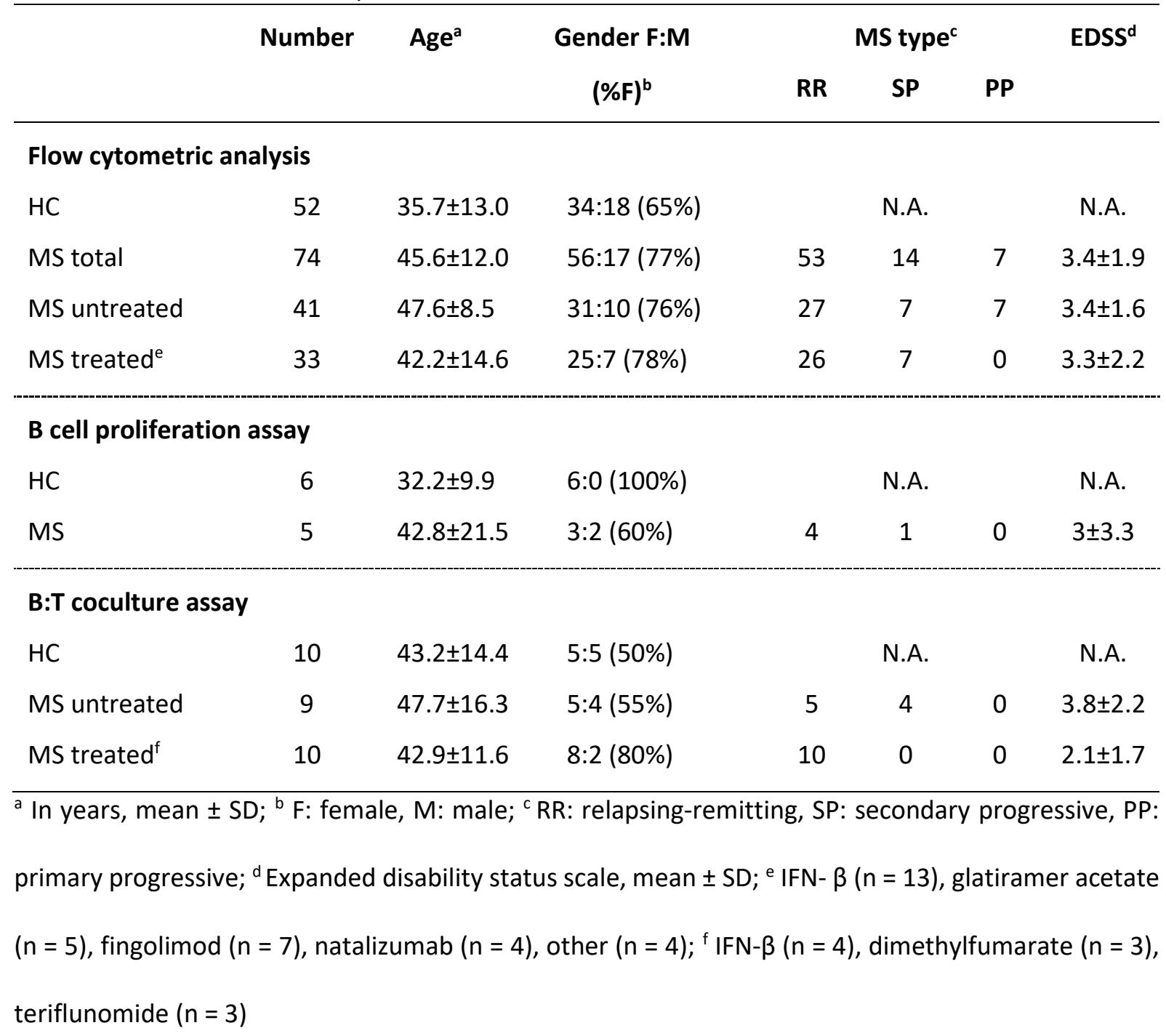




\section{Figure legends}

Figure 1. B cell expression of costimulatory and HLA molecules in the peripheral blood. (A) $C D 19^{+} B$ cells were gated from PBMC, followed by analysis of HLA-DR/DP/DQ, HLA-A/B/C, CD86, CD80 and CD40 expression based on isotype controls. (B-C) The percentage (B) and MFI (C) of HLA-DR/DP/DQ+, HLA$\mathrm{A} / \mathrm{B} / \mathrm{C}^{+}, \mathrm{CD} 86^{+}, \mathrm{CD} 80^{+}$and $\mathrm{CD} 40^{+} \mathrm{B}$ cells is depicted for $\mathrm{HC}(\mathrm{n}=52)$ and $\mathrm{MS}$ patients $(\mathrm{n}=74)$, including untreated $(n=41)$ and treated $(n=33)$ MS patients. Mean levels \pm SEM are shown. Mann-Whitney test and Kruskal-Wallis test of one-way ANOVA were used for statistics. ${ }^{*} p<0.05$

Figure 2. Expression of costimulatory and HLA molecules on B cells in the CNS. (A) Histograms of CD86 and CD80 expression in the CD19+ gate from paired PBMC and CSF cells. Results of 1 representative untreated MS patient are shown. (B-C) The percentage (B) and MFI (C) of HLA-DR/DP/DQ ${ }^{+}, H L A-A / B / C^{+}$, $\mathrm{CD}^{+} 6^{+}, \mathrm{CD} 80^{+}$and $\mathrm{CD} 40^{+} \mathrm{B}$ cells in paired PBMC and CSF cells was measured in $20 \mathrm{MS}$ patients. (D-E) MS brain tissue was stained for CD20, HLA-DR (D) or CD80 (E) and DAPI. A representative image of 1 out of 4 patients is shown. Scale bars are $50 \mu \mathrm{m}$. Mann-Whitney or Student's t-test were used for statistics. $* p<0.05, * * p<0.01, * * * p<0.001$

Figure 3. B cell proliferation towards viral and autoantigens in $H C(n=6)$ and MS patients $(n=5)$. (A) CFSE histograms of B cell proliferation in CM, under TT and CpG2006 stimulation. Results of 1 representative MS patient are shown. (B) Ratios of B cell expression (MFI) of CD80, HLA-DR and HLAA/B/C at day 13:day 0 following CpG2006 and antigen-specific stimulation of HC and MS patients. The mean ratio of expression (MFI) + SEM at day 13:day 0 is shown of total $C D 19^{+} B$ cells showing positive antigen-specific proliferation ( $\triangle P F>2)$ towards $T(n=3), C M V(n=2)$ or MBP $(n=2)$ in the white bars. As a control, upregulation of CD80 and HLA-DR expression following polyclonal CpG2006 stimulation $(n=4)$ is depicted in the grey bars. (C) The percentage of proliferating B cells against $T$, CMV, MBP and MOG stimulation after correction for background proliferation ( $\triangle \mathrm{PF}$ ). Gating was done on living cells in comparison to the negative control (CM). The cut-off for positive antigen-specific proliferation 
is indicated by the dashed line. Numbers of donors with positive antigen-specific proliferation are shown below the graphs. Due to insufficient B cell numbers, not all conditions could be included for all donors. Statistical analysis was performed using Mann-Whitney test. * $p<0.05$

Figure 4. T cell proliferation following B cell presentation of viral or CNS-specific antigens. Examples of proliferation histograms from 1 IFN- $\beta$ treated MS patient (A), 1 untreated MS patient (B) and $1 \mathrm{HC}$ (C) are given of total $\mathrm{T}$ cell proliferation $\left(\mathrm{CD}^{+}\right)$in cocultures of purified CFSE-labelled $\mathrm{T}$ cells and autologous purified B cells with the antigens or stimulus as indicated above the columns. CFSE ${ }^{-}$cells were gated within all T cells to include both $\mathrm{CD}^{+}$and $\mathrm{CD} 8^{+} \mathrm{T}$ cells. Percentages of $\mathrm{CFSE}$ proliferating $\mathrm{T}$ cells are indicated.

Figure 5. B cells from MS patients induce $T$ cell proliferation to viral and autoantigens by antigen presentation. (A) T cell proliferation ( $\triangle P F)$ towards PHA was measured for $\mathrm{HC}(n=5)$, untreated $(n=3)$ and treated $(n=5)$ MS patients. Due to insufficient cell numbers, PHA stimulation could not be included for all donors in the B:T coculture assay. The cut-off is indicated by the dashed line. (B) T cell proliferation $(\triangle P F)$ of $H C(n=10)$, untreated $(n=9)$ and treated $(n=10)$ MS patients induced by $B$ cell presentation of $\mathrm{TT}, \mathrm{CMV}, \mathrm{MBP}$ and MOG. Individual data and mean $\pm \mathrm{SEM}$ are plotted and the cut-off is indicated by the dashed line. Numbers of donors with positive antigen-specific proliferation are shown below the graphs. (C) The percentage of donors that was positive for T cell proliferation against TT, CMV, MBP or MOG. (D) Percentage blocking of $\mathrm{T}$ cell proliferation $(\triangle \mathrm{PF})$ following $\mathrm{B}$ cell presentation of TT using an anti-HLA-DR $(n=10)$, anti-HLA-A/B/C $(n=7)$ or isotype control $(n=5)$ antibody for combined untreated and treated MS patients and HC. Data are presented as mean + SEM. (E) Histograms showing T cell proliferation following B cell presentation of TT and blocking of the response by an anti-HLA-DR, anti-HLA-A/B/C or isotype control antibody for 1 representative treated MS patient. Statistics was done by Kruskal-Wallis test of one-way ANOVA, Chi-square test for contingency tables or Mann-Whitney test. $* p<0.05, * * * p<0.001$ 
Figure 6. Characterization of the B cell induced T cell response towards viral and CNS-specific antigens.

(A) $\mathrm{CD}^{+}$and $\mathrm{CD} 8^{+} \mathrm{T}$ cell proliferation following $\mathrm{B}$ cell presentation of $\mathrm{TT}, \mathrm{CMV}, \mathrm{MBP}$ or MOG. (B) Production of IFN- $\gamma$, IL-17A and IL-4 was measured in the supernatant of all conditions from the B:T coculture assay with positive T cell proliferation. All data are presented as mean + SEM. None of the $\mathrm{HC}$ demonstrated $\mathrm{B}$ cell induced $\mathrm{T}$ cell proliferation towards $\mathrm{MBP}$, hence the absence of cytokine production results (N.A.). Kruskal-Wallis test of one-way ANOVA was used for statistics. 